\title{
Gastrointestinal Stromal Tumor (GIST) Presenting as a Huge Pelvic Mass
}

\section{Raumforderung im Becken erweist sich als gastrointestinaler Stromatumor (GIST)}

Author

Affiliation
TH. Lee

Department of Obstetrics and Gynecology, Kosin University College of Medicine, Busan, Republic of Korea

\author{
Key words \\ - gastrointestinal stromal \\ tumors (GISTs) \\ - ovarian carcinoma \\ - pelvic mass \\ - sigmoid colon \\ Schlüsselwörter \\ - gastrointestinaler \\ Stromatumor (GIST) \\ - Ovarialkarzinom \\ - Raumforderung im Becken \\ - Sigma
}

\section{Abstract \\ $\nabla$}

Gastrointestinal stromal tumors (GISTs) are rare tumors that can arise anywhere in the tubular gastrointestinal tract. These tumors occasionally present as a pelvic mass, leading to a misdiagnosis of gynecological disease. A 47-year-old woman presented with a pelvic mass measuring $30 \mathrm{~cm}$ in diameter and highly elevated CA-125 levels. She underwent exploratory laparotomy with resection of the pelvic mass. The mass mimicking ovarian malignancy was found in the sigmoid colon and was correctly diagnosed as a GIST at surgery. It should be noted that preoperative diagnosis of GIST is uncommon, due to its rarity and the varying clinical presentation. However, non-gynecological tumors should be included in the differential diagnosis of a pelvic mass with atypical presentation. Gynecologists need to be cognizant of extra-ovarian pathology in patients presenting with an atypical pelvic mass.

\section{Introduction}

received 2.5.2012

revised $\quad 5.10 .2012$

accepted 28.10.2012

\section{Bibliography}

DOI http://dx.doi.org/

10.1055/s-0032-1328039

Geburtsh Frauenheilk 2013; 73:

70-73 ๑ Georg Thieme

Verlag KG Stuttgart · New York . ISSN 0016-5751

\section{Correspondence}

Prof. Taehwa Lee

Kosin University College

of Medicine

Department of Obstetrics

and Gynecology

34 Amnam-dong, Seo-gu

602-702 Busan

Republic of Korea

leehula@hanmail.net

\section{Zusammenfassung \\ $\nabla$}

Der gastrointestinale Stromatumor (GIST) ist ein seltener Tumor des Magen-Darm-Trakts. Diese Tumoren treten gelegentlich als Raumforderung im Becken auf, was zur Fehldiagnose eines Ovarialkarzinoms führen kann. Wir berichten hier von einer 47-jährigen Patientin mit einer Raumforderung im Becken mit einem Durchmesser von $30 \mathrm{~cm}$ und stark erhöhten CA-125-Werten. Sie wurde einer Laparotomie zu diagnostischen Zwecken unterzogen mit nachfolgender Resektion der Masse. Vor der Operation wurde die vorläufige Diagnose eines Ovarialkarzinoms gestellt. Bei der Operation wurde aber ein GIST im Sigma diagnostiziert. Es sei hier darauf hingewiesen, dass die präoperative Diagnostik gastrointestinaler Stromatumoren sehr schwierig ist, aufgrund der Seltenheit dieser Entität und der unspezifischen klinischen Präsentation. Es ist daher wichtig, auch andere, nicht ovarspezifische Pathologien in die Differenzialdiagnose einer Raumforderung im Becken einzubeziehen.

of GISTs are found incidentally during laparotomy, endoscopy, or other imaging studies. Around 15$50 \%$ of patients with GISTs have metastatic disease [6]. Because of their histology, GISTs presenting as pelvic masses can be mistaken for gynecological disease, such as ovarian tumor or uterine leiomyoma [7]. The majority of GISTs have diameters of less than $10 \mathrm{~cm}$. Large tumor size, increased mitotic rate, tumor necrosis, and a high Ki-67 index are all associated with malignancy and are accepted as the main prognostic factors for metastasis and mortality [8]. We report here on a case with an unusually large pelvic mass with the characteristics of a GIST. 

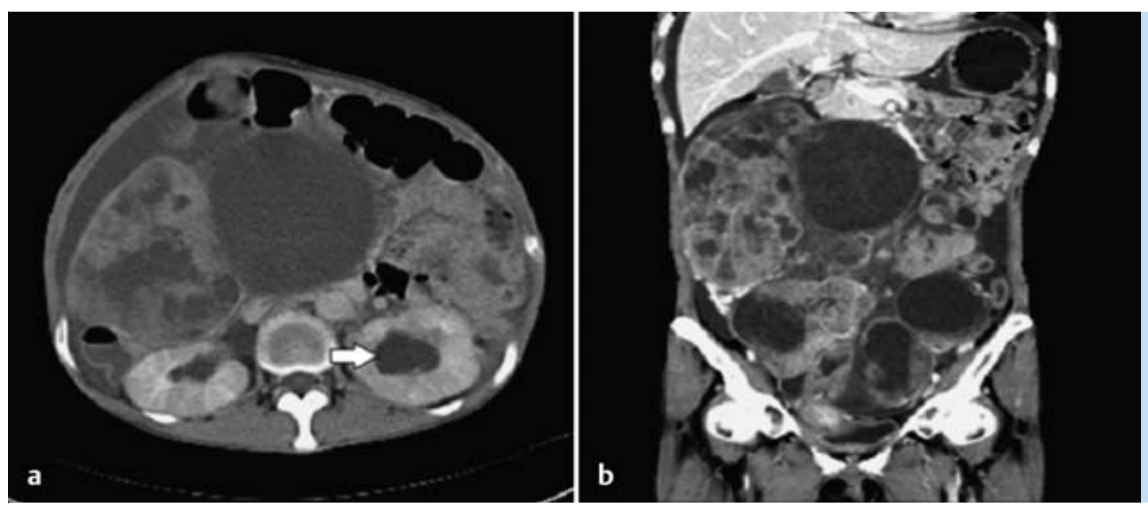

Fig. $1 \mathbf{a}$ and $\mathbf{b}$ Enhanced computerized tomography.

a Left-sided hydronephrosis (arrows) and a huge pelvic mass.

b Huge multiseptated mass occupying most of the abdominal cavity.
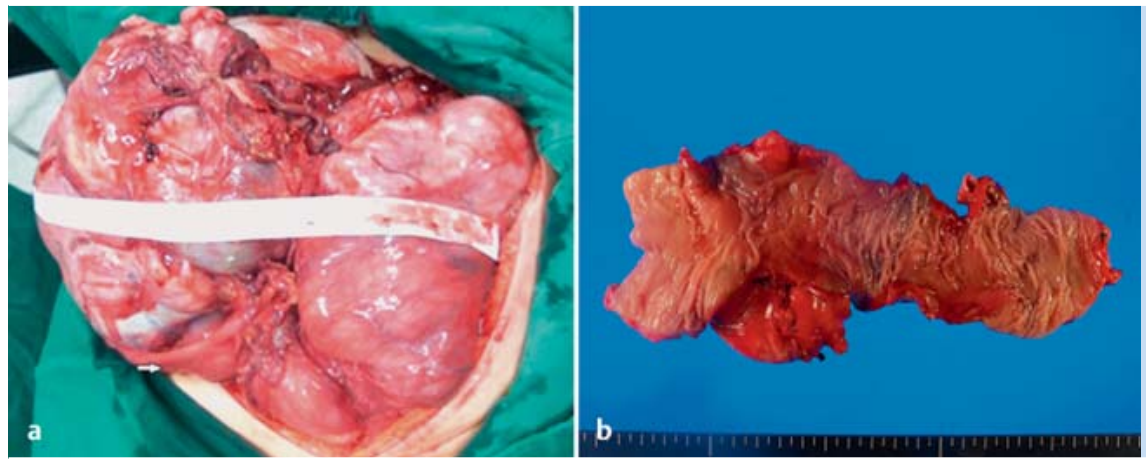

Fig. $2 \mathrm{a}$ and $\mathbf{b}$

a Gastointestinal stromal tumor during laparotomy, showing adherence of the sigmoid colon to the mass (arrows).

b Gross appearance after surgery. The tumor originated from sigmoid colon; however, the sigmoid lumen remained intact.

\section{Case Report}

A 47-year-old multiparous woman presented with a 2-year history of abdominal distension with encopresis. She described occasional dyspepsia and weight gain. She had ignored the mass until it was palpable, requiring her to present to hospital. The patient had noted that her bowel evacuations had increased to three times a day, and she also complained of vesical tenesmus. Her medical and surgical history was unremarkable, and her menstrual history was normal. She had had two spontaneous vaginal deliveries and two abortions. On physical examination, the patient appeared anemic. A large, round, firm, palpable mass resembling a late-term pregnancy was noted. CT scan of the abdomen revealed hydronephrosis of the left kidney, ascites, and a multiseptated mass occupying the entire abdomen, approximately $30 \times 20 \times 15 \mathrm{~cm}$ in size ( Fig. 1). However, CT scan did not show any bowel invasion. Serum CA-125 levels were $306 \mathrm{U} /$ $\mathrm{mL}$ (normal range $\leq 35 \mathrm{U} / \mathrm{mL}$ ), while serum levels for CA19-9 and CA15-3 were in the normal ranges. An initial diagnosis of ovarian cancer was made based on the presence of the large mass, ascites and elevated CA-125 levels, and the patient underwent surgery.

At surgery, a xypho-pubic incision was made, and the abdominal cavity was opened. The pelvis was completely filled by a large mass of apparently ovarian origin. The mass was adherent to the sigmoid colon, the ovaries and the posterior wall of the uterus ( Fig. 2). The patient underwent exploratory laparotomy with resection of the pelvic mass and sigmoid colectomy. Subsequently, the uterus and the adnexa were completely removed, together with $700 \mathrm{~mL}$ of bloody ascites. Macroscopically, the mass had a whitish-grey color, a diameter of $30 \mathrm{~cm}$, and contained hemorrhagic and necrotic areas ( $\bullet$ Fig. 2). A fragment of neoplastic tissue, similar to the mass, adhered to the posterior peritoneum. The uterus and omentum were intact; however, both ovaries were entangled with the mass, making them difficult to distinguish. No signs of metastasis to other sites were noted. Gross examination revealed that the mass originated from the sigmoid colon at the lumen of sigmoid colon, which remained intact ( Fig. 2). A final diagnosis of spindle-cell type GIST was made ( Fig. 3). After surgery, the patient had no complaints except
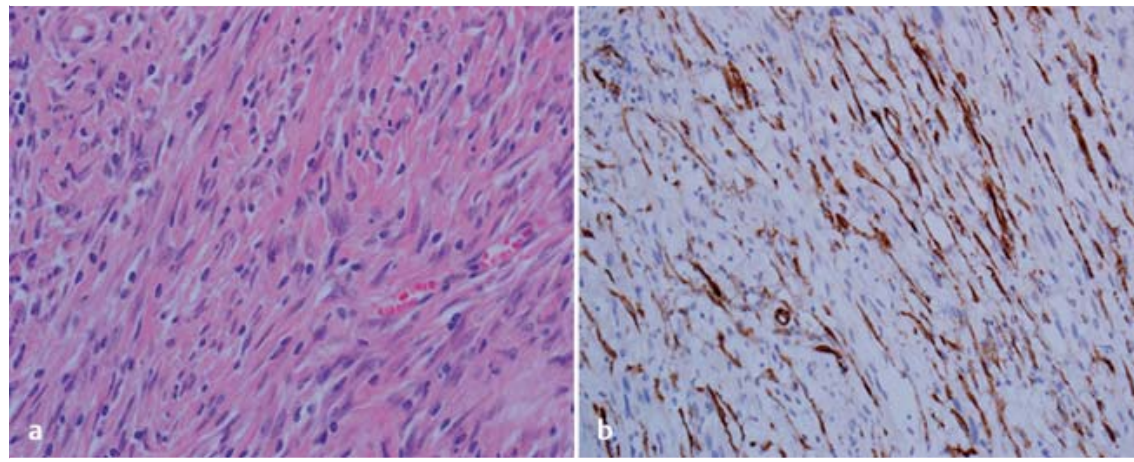

Fig. $3 \mathbf{a}$ and $\mathbf{b}$ Microscopic findings. a Spindled tumor cells in fascicles (hematoxylin and eosin stain; magnification $\times 100$ ). b c-kit positive immunostaining in the spindled areas (magnification $\times 40$ ). 
for frequent bowel urgency. The patient was treated with imatinib mesylate for two years on an outpatient basis. No signs of recurrence were noted on CT one year after surgery.

\section{Discussion \\ $\nabla$}

GISTs are mesenchymal tumors that arise mostly in the GI tract. They are more common in adulthood, with a peak incidence in the fifth and sixth decades of life and are seldom diagnosed in patients younger than 40 years of age [9]. In the present case, the GIST was found in a 47-year old patient. GISTs are most commonly located in the stomach and proximal small intestine, but can occur in any portion of the alimentary tract including the omentum, mesentery, and peritoneum [8], although, as reported here, GISTs may also have unusual origins from sites such as the sigmoid colon. Most patients with GISTs present with symptoms of varying severity, ranging from dysphagia, abdominal discomfort, altered bowel movements, and bowel obstruction, to acute or chronic gastrointestinal hemorrhage and peritonitis due to perforation [9]. In general, pain is dependent on tumor size. In the present case, the patient had a visible, palpable tumor and high CA-125 levels, and reported changes in bowel frequency as well as vesical tenesmus. Some authors have stratified tumors into risk categories based on their size and mitotic count. Tumors less than $2 \mathrm{~cm}$ in size with a mitotic count $<5 / 50$ in a high power field (HPF) are categorized as very low risk; tumors between 2$5 \mathrm{~cm}$ with a mitotic count $<5 / 50$ HPF are categorized as low risk; tumors $<5 \mathrm{~cm}$ in size with a mitotic count of 6-10/50 HPF or tumors with a size of 5-10 cm and a mitotic count of $<5 / 50 \mathrm{HPF}$ are categorized as intermediate risk; and tumors $>5 \mathrm{~cm}$ with a mitotic count of $>5 / 50 \mathrm{HPF}$, tumors greater than $10 \mathrm{~cm}$ and any mitotic rate, and tumors of any size with a mitotic rate $>10 / 50 \mathrm{HPF}$ are classified as high risk [10]. Our patient had a tumor greater than $30 \mathrm{~cm}$ and a mitotic count of more than 5/50 HPF, so we expected a poor prognosis. This case is particularly unusual because of the extremely large tumor size $(30 \times 20 \times 15 \mathrm{~cm})$; others authors have reported mean GIST sizes of $6-10.6 \mathrm{~cm}$ [4-8]. In addition, the tumor had a very high mitotic count of up to 8-9/50 HPF and, as mentioned previously, arose from the sigmoid colon.

Gastrointestinal stromal tumors rarely present as a pelvic mass and may be mistakenly diagnosed as ovarian tumors [11]. Preoperative diagnosis of a GIST is uncommon, due to their rarity, varying presentation, and the lack of distinguishing characteristics on imaging studies [10]. The diagnosis of GIST is based on cellular morphology and immunophenotype. Histologically, there are three types of GISTs: spindle cell (70\%), epithelioid (20\%), and mixed [10]. Approximately 95\% of GISTs carry an activating somatic mutation of CD117 (c-kit) [3]. CD117 is a transmembrane receptor tyrosine kinase encoded by the proto-oncogene c-kit located on chromosome 4 [12]. Positive immunohistochemical staining for CD117 is a defining characteristic of GISTs [12]. Tumor size and mitotic index are the two most important prognostic factors used for risk stratification of GISTs. Indeed, increased mitotic activity and large tumor size may be predictive of malignant degeneration. Outcomes in patients with GIST depend very much on clinical presentation and the histopathological features of the tumor. Thus, the overall 5-year survival rate for all patients with GIST ranges from $28-60 \%$, but this range can be stratified based on disease progression. For patients presenting with local- ized primary disease, the average survival rate is 5 years. For those presenting with metastatic or recurrent disease, the average survival rate is approximately $10-20$ months.

Although some authors have attempted to retrospectively review imaging studies of patients with GIST, the radiographic findings of these tumors are very nonspecific, so a preoperative presumptive diagnosis based on imaging is virtually impossible [2]. It is uncommon for patients with GIST to present with extensive ascites; however, some trace amounts of free fluid in the abdomen are not unusual. Patients may also present with peritonitis secondary to tumor rupture. Cytological examination of the peritoneal fluid obtained intraoperatively from our patient was negative for malignancy. Contrast-enhanced CT scan is the first choice imaging modality for patients with suspected abdominal mass, as it is useful for both preoperative staging and evaluation for metastatic disease. There are no specific CT findings for GIST tumors, although they typically appear as inhomogeneous masses with areas of necrosis and hemorrhage, with viable tumor areas showing contrast enhancement [13]. Positron emission tomography (PET) is not generally used for the evaluation of GISTs, although it may be useful in the early detection of tumor responsiveness to chemotherapy as well as to assess equivocal metastatic lesions [6]. Complete surgical resection is the treatment of choice, and biological therapy with imatinib mesylate is recommended for cases with incomplete resection or unresectable tumors, and when tumors have progressed to metastatic disease. As with ovarian cancer, complete tumor resection with rectosigmoidectomy is indicated for GISTs that directly invade the rectum or the sigmoid colon or disseminate into the pouch of Douglas [14]. In our case, we performed a debulking operation in addition to the sigmoidectomy, and treated the patient with oral imatinib mesylate in an outpatient setting. In adult patients with complete resection, the FDA has approved adjuvant therapy with imatinib for tumors with KIT-positive mutations. Although imatinib mesylate achieves a partial response or stable disease in the majority of GIST patients, complete and lasting response is rare; about half of the patients who initially benefit from imatinib mesylate treatment eventually develop drug resistance [1]. A more recent agent, sunitinib, which inhibits vascular endothelial growth factor receptor (VEGFR) in addition to KIT and platelet-derived growth factor receptor alpha (PDGFRA), has proven to be effective in patients intolerant or refractory to imatinib mesylate [1]. In summary, we report a case of a 47-year-old woman presenting with a pelvic mass and high serum CA-125 levels. Ultrasonography, CT, and other radiologic findings indicated ovarian cancer and the patient underwent surgery. Surgery revealed invasion of the sigmoid colon, so sigmoidectomy was performed and the mass was debulked. Histological examination subsequent to surgery confirmed the mass to be a GIST that had originated from the sigmoid colon. After surgery, the patient was treated with imatinib mesylate in an outpatient setting for two years without recurrence. Our case suggests that gynecologists should consider GIST in the differential diagnosis of patients presenting with an atypical pelvic mass.

\section{Acknowledgements \\ $\nabla$}

This report was supported by Kosin University of Medicine. 


\section{Conflict of Interest}

None.

\section{References}

1 Antonescu C. Gastrointestinal stromal tumors. Curr Top Microbiol Immunol 2011; Oct 21

2 Burkill GJ, Badran M, Al-Muderis $O$ et al. Malignant gastrointestinal stromal tumor: distribution, imaging features, and pattern of metastatic spread. Radiology 2003; 226: 527-532

3 Zighelboim I, Henao G, Kunda A et al. Gastrointestinal stromal tumours presenting as a pelvic mass. Gynaecol Oncol 2003; 91: 630-635

4 Pidhorecky I, Cheney RT, Kraybill WG et al. Gastrointestinal stromal tumors: current diagnosis, biologic behavior, and management. Ann Surg Oncol 2000; 7: 705-712

5 De Silva MVC, Reid R. Gastrointestinal stromal tumors (GIST): C-kit mutations, CD 117 expression, differential diagnosis and targeted cancer therapy with imatinib. Pathol Oncol Res 2003; 9: 13-19

6 Patil S, Jain S, Kaza RC et al. Giant gastrointestinal stromal tumor presenting as a palpable abdominal mass: an unusual presentation. ISRN Surg 2011; 2011: 894829
7 Morimura Y, Yamashita N, Koyama N et al. Gastrointestinal stromal tumor mimicking gynecological disease. Fukushima J Med Sci 2006; 52: 21-28

8 Matteo D, Dandolu $V$, Lembert $L$ et al. Unusually large extraintestinal GIST presenting as an abdomino-pelvic tumor. Arch Gynecol Obstet 2008; 278: 89-92

9 Wingen CB, Pauwels PA, Debiec-Rychter M et al. Uterine gastrointestinal stromal tumour (GIST). Gynecol Oncol 2005; 97: 970-972

10 Fletcher CD, Berman JJ, Corless C et al. Diagnosis of gastrointestinal stromal tumors: a consensus approach. Int J Surg Pathol 2002; 10: 81-89

11 Carlomagno G, Beneduce P. A gastrointestinal stromal tumor masquerading as an ovarian mass. World J Surg Oncol 2004; 2: 15

12 Dorfman DM, Bui MM, Tubbs RR et al. The CD117 immunohistochemistry tissue microarray survey for quality assurance and interlaboratory comparison: a College of American Pathologists Cell Markers Committee Study. Arch Pathol Lab Med 2006; 130: 779-782

13 Da Ronch T, Modesto A, Bazzocchi M. Gastrointestinal stromal tumour: spiral computed tomography features and pathologic correlation. Radiol Medi 2006; 111: 661-673

14 Takahashi O, Sato N, Miura Y et al. Surgical indications for combined partial rectosigmoidectomy in ovarian cancer. J Obstet Gynaecol Res 2005; 31: 556-561 